\title{
Effect of structurally-induced lateral confinement on split Hopkinson pressure bar test specimens of concrete-like materials
}

\author{
E.A. Flores-Johnson ${ }^{1}$ and Q.M. Li ${ }^{2}$, a \\ ${ }^{1}$ School of Civil Engineering, The University of Sydney Building J05, Sydney NSW 2006, Australia \\ ${ }^{2}$ School of Mechanical, Aerospace and Civil Engineering, The University of Manchester, Manchester M13 9PL, UK
}

\begin{abstract}
In dynamic testing of concrete-like materials, there is a need in distinguishing structural effects from genuine strainrate effects. In this paper, this generic problem is studied by numerical simulations based on a phenomenological material model available in the commercial finite element (FE) code Abaqus. The numerical results show that the increase of the dynamic increase factor (DIF) with the increase of strain-rate in concrete-like materials in a Split Hopkinson Pressure Bar (SHPB) test is a phenomenon related not only to material strain-rate effects but also to structural effects. It was found that dilation, surface friction and lateral inertia cause lateral confinement, which enhances DIF when the strain-rate is greater than a transition strainrate in the order of $10^{2} \mathrm{~s}^{-1}$. Although, genuine strain-rate effect may exist as suggested by meso-scale simulations in previous investigations, the findings in this study show that structural effects have a significant contribution to the increase of DIF, and therefore, it is necessary to correctly calibrate existing phenomenological models and interpret the results obtained from split Hopkinson pressure bar (SHPB) tests.
\end{abstract}

\section{Introduction}

It is well-known that compressive properties of concrete and similar brittle materials, e.g., mortar or rock, exhibit strain-rate dependence [1-3]. The increase of the compressive strength in dynamic testing is commonly described by using the dynamic increase factor (DIF), which is defined by the ratio of the dynamic strength to the quasi-static strength in unconfined uniaxial compression [4]. The DIF of concrete-like materials in the high strain-rate range of $10^{1}-10^{3} \mathrm{~s}^{-1}$ is often obtained by performing dynamic test using a split Hopkinson pressure bar (SHPB); however, it has long been debated whether the increase of compressive strength observed in SHPB tests of unconfined specimens is an intrinsic material property or is related to structural or inertial effects [4-6]. It has been shown that the induced lateral confinement in SHPB test, which causes increase of DIF, may be caused by friction between the bars and the specimen [5,7], large specimen diameters [8,9], and radial inertia [10,11]. The enhanced DIF observed when the induced lateral confinement is present should not be related to material property and the SHPB data should be correctly interpreted.

Some recent investigations using meso-scale modelling have shown that the enhancement of mechanical properties in dynamic testing is also related to intrinsic material properties. A recent study by Snozzi et al. [12] shows than the increase of strength in concrete with the increase of strain-rate is associated with the fast extension of micro-cracks, which is related to the intrinsic opening time in the cohesive law used to define the interface strength between mortar and aggregates. Qin and

\footnotetext{
${ }^{a}$ Corresponding author: qingming. li@manchester .ac.uk
}

Zhang [13] modelled dynamic compression of concrete using the particle element method. They observed the formation of reticular and dispersed cracks at high strainrate and related the increase of strength to higher force chain intensity and oblique bifurcations of the force chain distribution. In their study, the increase of strength was also attributed to higher frictional forces due to the increase of contact bond breaks [13]. Both studies agree with experiments reported by Ross [14] where crack pattern, density and distribution increase with the increase of strain-rate. Song and Lu [15] reported that the increase of DIF with strain-rate is related to the heterogeneity of multiphase materials.

Although meso-scale models bring a better understanding of the mechanisms responsible for the increase of DIF with strain-rate in concrete-like materials, they are impractical for the macroscopic modelling of large engineering structures subjected to dynamic actions, e.g. blast and impact. In this case, a computationallyeffective continuum model is needed for the macroscopic response. However, the phenomenological models should be able to capture material's mesoscopic and microscopic behaviours using parameters that have clear physical meanings.

Dilatancy of concrete-like materials refers to the volume increase with shear stress, which may result from the formation and growth of micro-cracks in compression $[1,16]$. This suggests that dilation may be related to the increase of DIF in concrete-like materials in a SHPB test because of the lateral confinement due to the volume increase. A recent numerical study by Elmer VII et al. [17] showed that the enhancement of concrete strength in SHPB tests is strongly related to shear dilation. They investigated the influence of the dilation parameter of the $\mathrm{K} \& \mathrm{C}$ model 
Table 1. Geometrical details, material properties and material model parameters.

\begin{tabular}{|l|c|c|}
\hline $\begin{array}{l}\text { Material } \\
\text { properties }\end{array}$ & Specimen [3] & $\begin{array}{c}\text { Input/output } \\
\text { bar [4] }\end{array}$ \\
\hline Density $\rho\left(\mathrm{kg} / \mathrm{m}^{3}\right)$ & 2000 & 7800 \\
\hline Young's modulus $E(\mathrm{GPa})$ & 20 & 210 \\
\hline Poisson's ratio $v$ & 0.2 & 0.3 \\
\hline Friction angle $\beta\left(^{\circ}\right)$ & $0,30,50$ & - \\
\hline Dilation angle $\psi\left({ }^{\circ}\right)$ & $0,30,50$ & - \\
\hline Geometrical details & & \\
\hline Diameter $D(\mathrm{~mm})$ & 12,20 & 20 \\
\hline Length $L(\mathrm{~mm})$ & 6 & 1000 \\
\hline
\end{tabular}

available in LS-DYNA and found that the DIF of concrete increases when the dilation parameter is increased.

As aforementioned, there is a need to improve our understanding of structural effect contributions to the observed strain-rate effects using phenomenological material models, but nonetheless with meaningful parameters that can be easily determined for engineering numerical simulations. In this paper, this problem is addressed by presenting numerical simulations dealing with the effect of induced lateral confinement using a phenomenological material model available in the FE code Abaqus.

This work focuses on a numerical study of a material model that takes into account dilation for continuum finite element modelling. A parametric study is performed to further investigate the effect of dilation, friction angle, friction coefficient and specimen dimensions on the increase of DIF of concrete-like materials in SHPB tests. FE simulations of SHPB test using Abaqus/Explicit employs the Extended Drucker-Prager material model. In all numerical simulations, strain-rate enhancement factor is not employed in order to study only structural effects. FE models are described in Sect. 2. Numerical results and discussion are presented in Sect. 3, which are followed by conclusions in Sect. 4.

\section{Numerical simulations of SHPB tests}

The FE simulations of SHPB tests in this work were performed with a similar setup as that presented by Grote et al. [3]. The geometrical details of the specimen, input and output bars are depicted in Table 1. The numerical simulations were performed using the commercial (FE) software Abaqus/Explicit [18]. To reduce computational costs, a representative quarter-model was constructed using 8-node brick elements with reduced integration (Fig. 1). The elements of the specimen and the elements in the impact areas of the bars (Fig. 1) have an average size of $0.2 \times 0.2 \times 0.2 \mathrm{~mm}^{3}$. The total number of elements in a $12-\mathrm{mm}$ specimen is 17250 , while it is 43365 for each bar. A mesh sensitivity analysis showed that smaller element size did not provide an increase in accuracy.

The input and output bars of the SHPB were modelled using an isotropic elastic material model with input parameters showed in Table 1 . The specimen was modelled in Abaqus/Explicit using the extended DruckerPrager material model with a linear yield criterion with parameters showed in Table 1 and hardening input curve

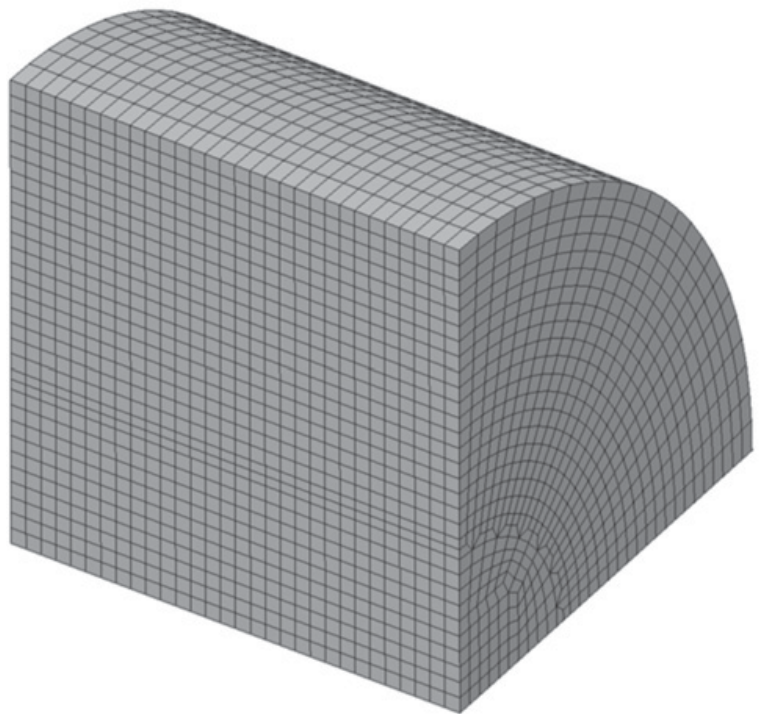

Figure 1. Finite element mesh of the split Hopkinson pressure bar test specimen.

showed in Fig. 2. An overview of the model is described in the next section.

\subsection{Extended Drucker-Prager constitutive model}

The extended Drucker-Prager material with linear yield criterion is given by [18],

$$
F=t-p \tan \beta-d=0
$$

where $\beta$ is friction angle of the material, which is the slope of the linear yield surface in the $p-t$ stress plane. The cohesion of the material $d$ is given by $d=$ $\sigma_{c}(1-(1 / 3) \tan \beta)$, where $\sigma_{c}$ is the compressive yield stress. The deviatoric stress measure $t$ is defined as,

$$
t=\frac{1}{2} q\left[\frac{1}{K}-\left(1-\frac{1}{K}\right)\left(\frac{r}{q}\right)^{3}\right]
$$

where $q$ is the Mises equivalent stress, $r$ is the third invariant of the deviatoric stress and $K$ is the ratio of the yield stress in triaxial tension to the yield stress in triaxial compression [18]. The parameter $K$ has to be within the range $0.788 \leq K \leq 1$ to ensure the convexity of the yield surface. The flow rule of the extended Drucker-Prager model is given by,

$$
d \boldsymbol{\varepsilon}^{p}=\frac{d \boldsymbol{\varepsilon}^{p}}{c} \frac{\partial G}{\partial \boldsymbol{\sigma}}
$$

where $\boldsymbol{\varepsilon}^{p}$ is the equivalent plastic strain, $c$ is a constant given by $c=1-(1 / 3) \tan \psi$ if hardening is defined in uniaxial compression and $\sigma$ is the stress tensor. The flow potential $G$ is defined as,

$$
G=t-p \tan \psi
$$

where $p$ is the equivalent pressure stress and $\psi$ is the dilation angle. If $\psi=0$, the plastic deformation is incompressible and when $\psi \geq 0$ the material dilates. 


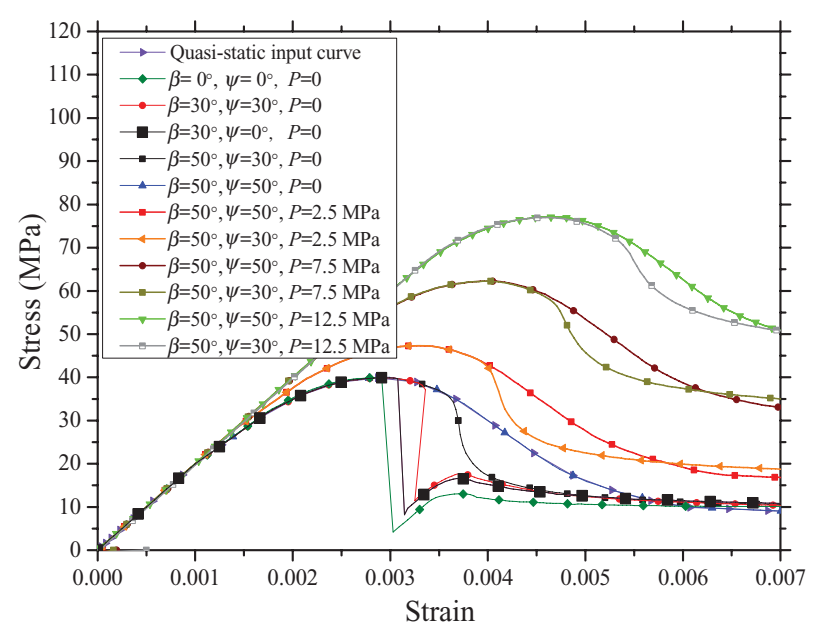

Figure 2. Stress-strain curves for different input parameters and lateral confinements (P) at $\dot{\varepsilon}=8 \times 10^{-2} \mathrm{~s}^{-1}$ for the DruckerPrager model.

When $\beta=\psi$ and $K=1$, a fully associated flow is obtained and the original Drucker-Prager model is reinstated [18]. For concrete-like materials, it has been found that $\beta$ and $\psi$ are in the ranges of $20-60^{\circ}$ and 20 $50^{\circ}$, respectively [19-21].

\subsection{Numerical analyses of SHPB test}

A parametric study comprising the effects of the friction angle, dilatancy, friction coefficient and specimen diameter on the DIF of concrete subjected to strain-rates in the range of $10^{1}-10^{3} \mathrm{~s}^{-1}$ is performed. For the numerical simulations, three friction angle values $\beta\left(0^{\circ}, 30^{\circ}, 50^{\circ}\right)$, three dilation angles values $\psi\left(0^{\circ}, 30^{\circ}, 50^{\circ}\right)$ and three friction coefficient values $\mu(0,0.1,0.2)$ are used.

To achieve different strain-rates, trapezoidal stress pulses with different rising time and intensity are applied to the end of the incident bar. The rising time varies from 60 to $200 \mu$ s while the duration and unloading times are fixed to $20 \mu \mathrm{s}$ as they do not have significant effect on the strain-rate and only strain at strength is of interest in this study. The intensity of the pulse varies from 50 to $900 \mathrm{MPa}$.

No strain-rate enhancement factor is employed in both material model and software in order to study only structural effects. To measure the axial stress and strain rate in the specimen in the numerical simulations, the formulae described by $\mathrm{Lu}$ and $\mathrm{Li}$ [22] are used. These formulae use the nodal forces and velocities at the specimen interfaces to obtain the time histories of the average engineering stress $\sigma(t)$ and average strain-rate $\dot{\varepsilon}(t)$ over the specimen, i.e.,

$$
\begin{gathered}
\sigma(t)=\frac{P_{I}(t)+P_{O}(t)}{2 A_{S}} \\
\dot{\varepsilon}(t)=\frac{V_{I}(t)-V_{O}(t)}{L_{S}}
\end{gathered}
$$

where $P_{I}(t)$ and $V_{I}(t)$ are the force and velocity time histories at the interface between the input bar and the specimen, respectively; and $P_{O}(t)$ and $V_{O}(t)$ are the force and velocity time histories at the interface between the (a)

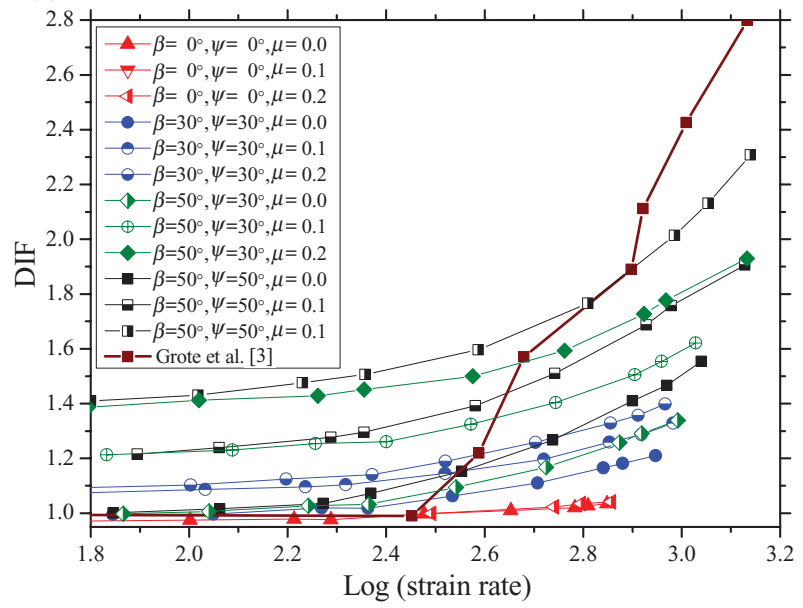

(b)

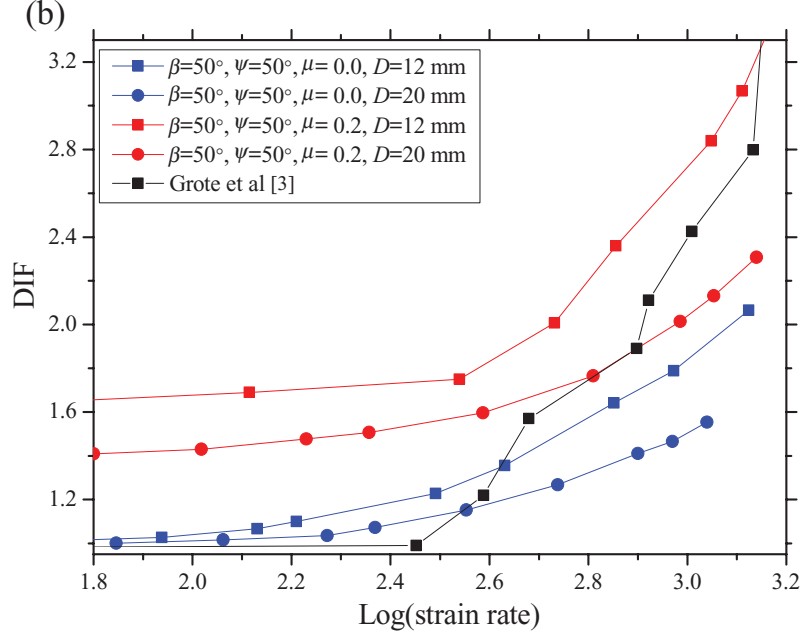

Figure 3. Numerical results of SHPB test using different parameters: (a) Effect of dilation parameter $\beta$, dilation angle $\psi$ and friction coefficient $\mu$; (b) Effect of specimen diameter $D$.

output bar and the specimen, respectively. The constant $A_{S}$ is the cross-sectional area of the specimen and $L_{S}$ is the specimen length.

It is known that if the peak stress in the specimen is reached before the stress equilibrium is reached, the test may not be valid. Therefore, for all the simulations, the dynamic stress equilibrium was verified using the parameter $R(t)$ defined as [22,23],

$$
R(t)=2\left|\frac{P_{I}(t)-P_{O}(t)}{P_{I}(t)+P_{O}(t)}\right| .
$$

It is generally accepted that when there is difference of $5 \%$ or less in stress across the SHPB specimen with respect to the average stress, i.e., $R(t) \leq 0.05$, the equilibrium has been reached [24].

\section{Numerical results and discussion}

Figure 2 shows quasi-static compression stress-strain curves performed at low strain-rate $\left(\dot{\varepsilon}=8 \times 10^{-2} \mathrm{~s}^{-1}\right)$. It can be seen that although parameters $\beta$ and $\psi$ define the shape of the softening part of the curves as expected, these parameters have negligible influence on the 


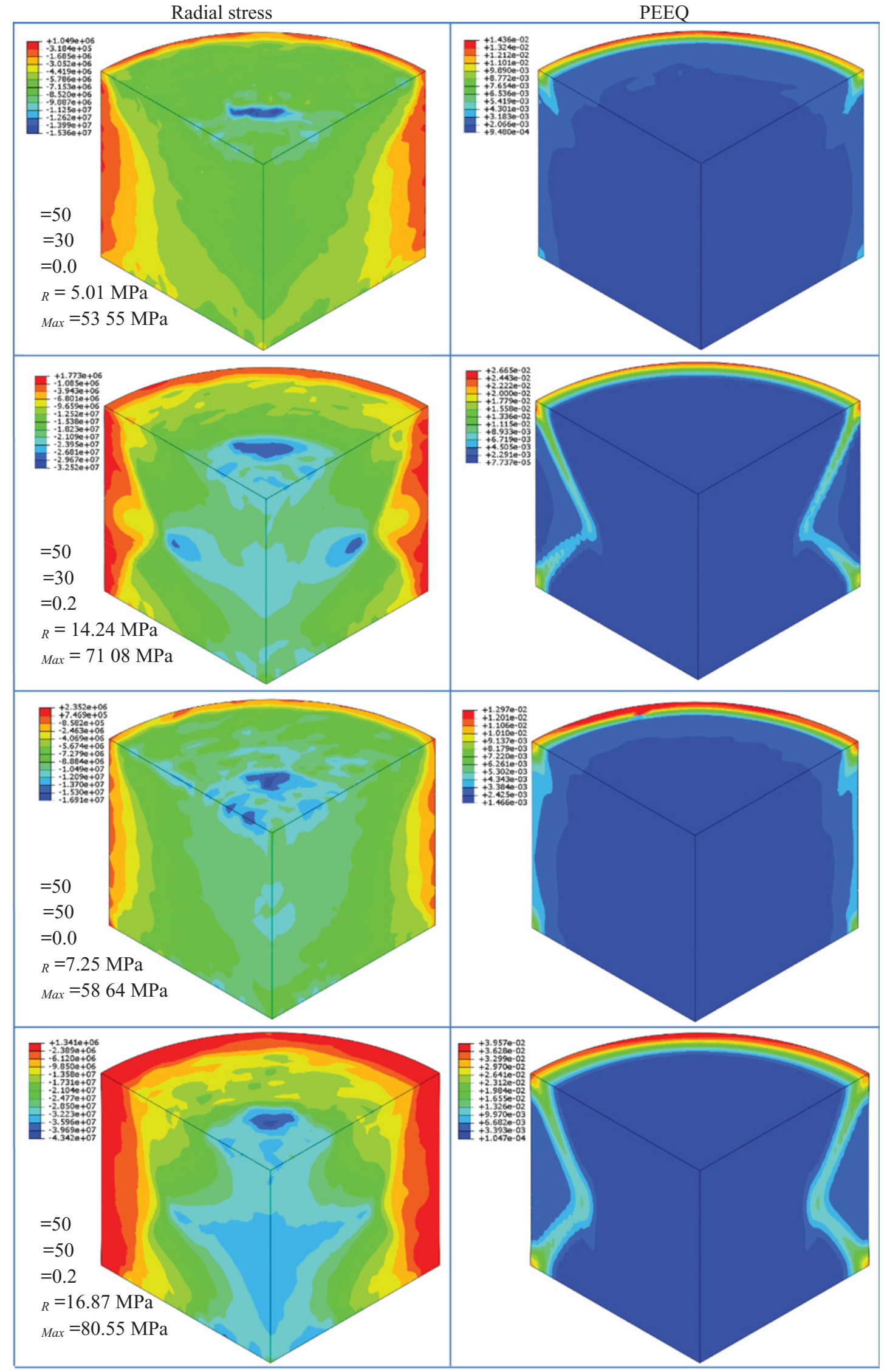

Figure 4. Contour plots of radial stress and PEEQ for a $\log$ (strain-rate $)=3$ for various material parameters sets. 
compressive strength, which remains constant at $40 \mathrm{MPa}$. However, it can be seen in Fig. 3(a) (SHPB simulations) that parameters $\beta$ and $\psi$ largely influence the dynamic response of mortar as indicated by the increase of DIF when the strain-rate increases, particularly for strain-rates higher than $10^{2} \mathrm{~s}^{-1}$. This results indicate that a good calibration of parameters $\beta$ and $\psi$ should be performed when strain-rates higher than $10^{2} \mathrm{~s}^{-1}$ are simulated.

The effect of friction on the dynamic response in SHPB tests is well-known, which leads to errors in the DIF $[4,25,26]$, particularly in concrete-like materials due to their coarser surface when compared to metals [27] and therefore friction should not be completely neglected even though lubricants are used. Figure 3(a) shows the results of the effect of the friction coefficient $\mu$ on the dynamic response of mortar for various $\beta$ and $\psi$. It can be seen that when $\beta=\psi=0$, the increase of $\mu$ does affect the dynamic response of the material; however when $\beta$ and $\psi$ increase, the DIF increases with the increase of $\mu$. This can be explained by the fact that when $\beta$ increases the material becomes more pressure dependant, and therefore, more sensitive to the induced lateral confinement due to the friction and dilation. Results from Grote et al. [3] are included in Fig. 3 for comparison. It can be seen that when $\beta=\psi=50^{\circ}$ and $\mu=0.2$ the numerical predictions are closer to the experimental results; however, this value is very large because $\mu \approx 0.05-0.1$ when lubricants are used in SHPB tests. Unfortunately, the friction coefficient is not given in Grote et al. [3] neither it is mentioned if lubricants were used. It is believed that the increase of DIF shown in Grote et al. [3] is partially due to genuine strain-rate effects and partially due to structural effects. Figure 4 shows contour plots of radial stress and equivalent plastic strain (PEEQ) for specimens at high strain-rates of approximately $10^{3} \mathrm{~s}^{-1}$ for various sets of $\beta, \psi$ and $\mu$. It can be observed that the lateral confinement, which can be expressed by the compressive radial stresses, increases when the friction coefficient is increased as expected. It can also be seen that for specimens with same interface friction coefficient, the lateral confinement increases when the dilation parameter is increased, and the rate of the increase is higher for higher strain-rates.

From Fig. 2, it can be seen that the peak stresses due to the lateral confinement $P$ are somewhat comparable with the peak stresses in Fig. 4 for the levels of average radial stresses that act as lateral confinement in the samples. It is observed that PEEQ also increases when the dilation parameter is increased (for example, in Fig. 4, compare the PEEQ contours when $\psi=30^{\circ}$ and $\mu=0.2$, with the PEEQ contours when $\psi=50^{\circ}$ and $\mu=0.2$ ). This confinement effect is produced in the hourglass-shaped zone showed in Fig. 5, which is delimited by the fault regions with high PEEQ and volumetric expansion or dilation. This phenomenon has been observed by Elmer VII et al. [17] in numerical simulations using the $\mathrm{K} \& \mathrm{C}$ model in LS-DYNA. It is worth mentioning that the dilation parameter is crucial in the numerical prediction of concrete strength particularly for strain-rates higher than $10^{2} \mathrm{~s}^{-1}$ and a good calibration of this parameter should be performed to avoid an over prediction of strength when strain-rate effect is incorporated into the model.

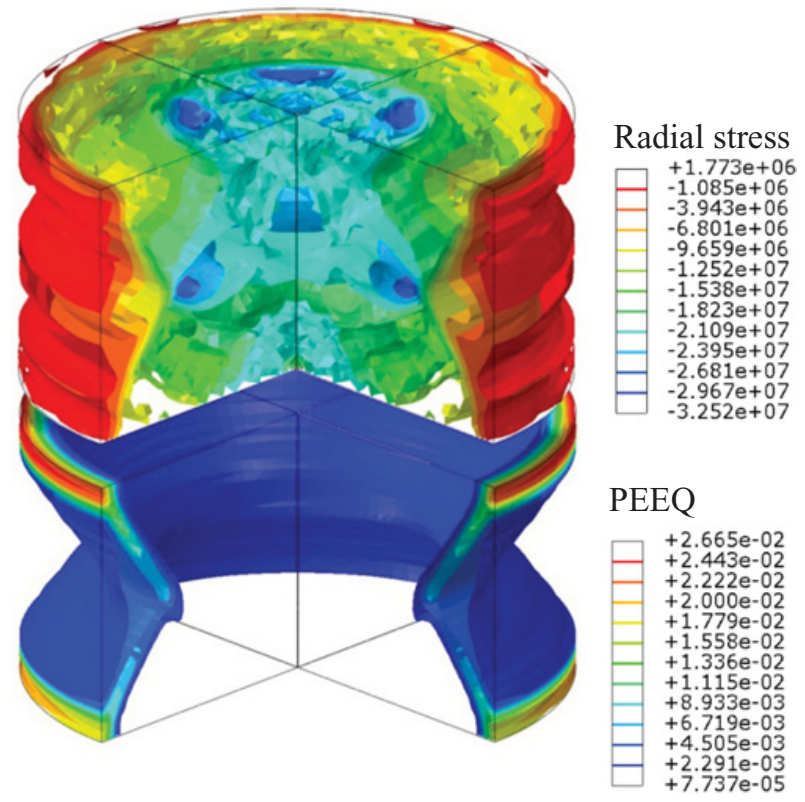

Figure 5. Contour plots of radial stress and PEEQ for a $\log ($ strain-rate $)=3$ and $\beta=50^{\circ}, \psi=30^{\circ}$ and $\mu=0.2$.

Figure 3(b) shows numerical simulations for specimens with diameters of $12 \mathrm{~mm}$ and $20 \mathrm{~mm}$. It can be seen that an increase in the specimen diameter while keeping the same length $(6 \mathrm{~mm})$ leads to an increase of DIF which agrees with previous research $[8,27]$. This clearly demonstrates that inertia has an effect on the dynamic response, which should not be considered as genuine strain-rate effect.

\section{Conclusions}

The numerical study conducted in this paper shows that the increase of the dynamic increase factor (DIF) with strainrate for concrete-like materials in SHPB tests is a complex phenomenon related not only to material strain-rate effects but also to structural effects. It is demonstrated that several factors including dilation, friction coefficient and lateral inertia may contribute to lateral confinement, which is the main mechanism in the increase of DIF, particularly in the strain-rate range of $10^{2}-10^{3} \mathrm{~s}^{-1}$. Although, some genuine strain-rate effects may exist as suggested by mesoscale simulations in previous investigations, the findings in this study show that structural effects have a significant contribution to the increase of DIF. Therefore, an effort to correctly calibrate existing phenomenological models and interpret the results obtained from SHPB tests should be done to include genuine strain-rate effect on DIF in continuum macroscopic models.

\section{References}

[1] P. Bischoff, S. Perry, Mater. Struct. 24, 425 (1991).

[2] C.A. Ross, J.W. Tedesco, S.T. Kuennen, ACI Mater. J. 92 (1995).

[3] D.L. Grote, S.W. Park, M. Zhou, Int. J. Impact Eng. 25, 869 (2001). 
[4] Q.M. Li, H. Meng, Int. J. Solids Struct. 40, 343 (2003).

[5] J.F. Georgin, J.M. Reynouard, Cem. Concr. Compos. 25 (2003).

[6] P. Rossi, F. Toutlemonde, Mater. Struct. 29 (1996).

[7] M.M. Al-Mousawi, S.R. Reid, W.F. Deans, Proc. Inst. Mech. Eng. Part C: J. Mech. Eng. Sci. 211 (1997).

[8] M. Zhang, H.J. Wu, Q.M. Li, F.L. Huang, Int. J. Impact Eng. 36, 1327 (2009).

[9] X.Q. Zhou, H. Hao, Int. J. Solids Struct. 45 (2008).

[10] G. Cusatis, Int. J. Impact Eng. 38, 162 (2011).

[11] Z.C. Mu, A.N. Dancygier, W. Zhang, D.Z. Yankelevsky, Int. J. Impact Eng. 49, 91 (2012).

[12] L. Snozzi, A. Caballero, J.F. Molinari, Cem. Concr. Res. 41 (2011).

[13] C. Qin, C. Zhang, Int. J. Impact Eng. 38 (2011).

[14] C.A. Ross, Crack patters resulting from high strainrate tests on concrete, 1992, Air Force Engineering and Services Center: Tyndall AFB, Florida, USA.

[15] Z. Song, Y. Lu, Int. J. Impact Eng. 46 (2012).

[16] C. Noble, E. Kokko, I. Darnell, T. Dunn, L. Hagler, L. Leininger, Concrete model descriptions and summary of benchmark studies for blast effects simulations, 2005, Lawrence Livermore National Laboratory (LLNL), Livermore, CA.

[17] W. Elmer VII, E. Taciroglu, L. McMichael, Int. J. Impact Eng. 45, 1 (2012).

[18] Simulia, Abaqus Analysis User's Manual (Version 6.11), 2011, SIMULIA.

[19] S.W. Park, Q. Xia, M. Zhou, Int. J. Impact Eng. 25, 887 (2001).

[20] J.-F. Jiang, Y.-F. Wu, Int. J. Solids Struc. 49, 445 (2012).

[21] B.M. Luccioni, M. Luege, Int. J. Impact Eng. 32, 1248 (2006).

[22] Y.B. Lu, Q.M. Li, Int. J. Protective Struct. 1, 363 (2010).

[23] G. Ravichandran, G. Subhash, J. Am. Ceram. Soc. 77, 263 (1994).

[24] B. Song, W. Chen, Lat. Am. J. Solids Struct. 2, 113 (2005).

[25] R.S. Hartley, T.J. Cloete, G.N. Nurick, Int. J. Impact Eng. 34, 1705 (2007).

[26] D.J. Kim, K. Sirijaroonchai, S. El-Tawil, A.E. Naaman, Int. J. Impact Eng. 37, 141 (2010).

[27] Q.M. Li, Y.B. Lu, H. Meng, Int. J. Impact Eng. 36, 1335 (2009). 